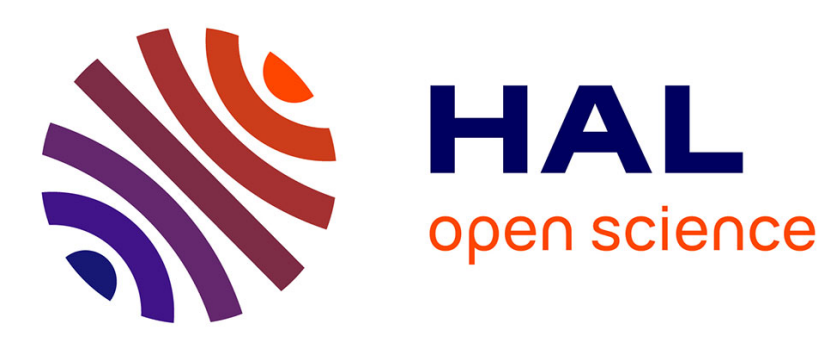

\title{
La discrimination positive territoriale: de l'égalité des chances à la mixité urbaine
}

Thomas Kirszbaum

\section{To cite this version:}

Thomas Kirszbaum. La discrimination positive territoriale: de l'égalité des chances à la mixité urbaine . Pouvoirs - Revue française d'études constitutionnelles et politiques, 2004, pp.101-118. halshs01100424

\section{HAL Id: halshs-01100424 \\ https://shs.hal.science/halshs-01100424}

Submitted on 6 Jan 2015

HAL is a multi-disciplinary open access archive for the deposit and dissemination of scientific research documents, whether they are published or not. The documents may come from teaching and research institutions in France or abroad, or from public or private research centers.
L'archive ouverte pluridisciplinaire HAL, est destinée au dépôt et à la diffusion de documents scientifiques de niveau recherche, publiés ou non, émanant des établissements d'enseignement et de recherche français ou étrangers, des laboratoires publics ou privés. 


\section{THOMAS KIRSZBAUM}

\section{LA DISCRIMINATION POSITIVE TERRITORIALE : DE L'ÉGALITÉ DES CHANCES À LA MIXITÉ URBAINE*}

U FIL DE SON HISTOIRE, LA SOCIÉTÉ FRANÇAISE a intégré des immigrés de diverses origines. Si, pour une majorité d'entre eux, l'assimilation culturelle demeure une réalité ${ }^{1}$, les immigrés et leurs descendants qui résident dans les quartiers «relégués» se heurtent à de multiples obstacles venant entraver leur mobilité sociale. Ces obstacles, qui concernent surtout les populations originaires d'Afrique du Nord et sub-saharienne et de Turquie, sur-représentés dans ces quartiers, ne s'expliquent pas seulement par une appartenance plus fréquente aux catégories sociales désavantagées, mais aussi par des conditions de vie urbaine et des discriminations qui redoublent l'inégalité des chances. Un récent rapport du Conseil d'analyse économique a souligné les fortes inégalités dont ces populations sont victimes dans l'accès à différents marchés urbains (éducation, emploi, formation, logement...). Selon ce rapport, leur déficit d'intégration socio-économique expliquerait le repli d'une partie des habitants des «zones urbaines sensibles» sur leurs spécificités culturelles et religieuses ${ }^{2}$.

\footnotetext{
* Ce texte a fait l'objet de discussions avec Daniel Sabbagh et Renaud Epstein, ici remerciés.

1. Voir Michèle Tribalat (dir.), De l'immigration à l'assimilation. Enquête sur les populations d'origine étrangère en France, La Découverte, 1996.

2. Jean-Paul Fitoussi et al., Ségrégation urbaine et Intégration sociale, rapport du Conseil d'analyse économique, La Documentation française, 2004.
} 
Ces constats interrogent l'efficacité de près de trente années d'une «politique de la ville» qui constitue de facto la principale politique publique d'intégration des immigrés. Certes, il existe en France une politique d'intégration, intitulée comme telle, seule politique catégorielle destinée aux immigrés. Mais ses objectifs et financements ont fini par se confondre avec ceux de la politique de la ville ${ }^{3}$, du moins jusqu'au récent recentrage du FASILD ${ }^{4}$ sur l'accueil des primo-arrivants et la lutte contre les discriminations. Les critères de sélection des «quartiers cibles» de la politique de la ville ont été définis de telle manière que sa géographie d'intervention inclut prioritairement (mais pas uniquement) les lieux de concentration des immigrés et de leurs descendants. Les indicateurs retenus, à partir de 1991, pour la sélection de ces quartiers (proportion de chômeurs de longue durée, de jeunes de moins de

10225 ans et d'étrangers, ce dernier critère ayant été remplacé, en 1995, par celui des non-diplômés qui englobe les enfants d'étrangers devenus français) assurent en effet la prise en compte de ces groupes.

Le choix de ces critères pourrait laisser penser que le territoire n'a d'autre fonction, dans la politique de la ville, que de cibler des groupes ethniques que le modèle universaliste français interdit d'identifier pour l'attribution d'un avantage catégoriel. Ainsi, il est devenu courant de voir dans la politique de la ville une formule de «discrimination positive territoriale» consistant à «donner plus aux territoires qui ont moins » et, de manière indirecte, aux populations qui y résident ${ }^{5}$. L'hypothèse a même été avancée d'un «agenda caché» de la politique de la ville qui pratiquerait une "affirmative action territoriale» afin d'éviter les effets de stigmatisation associés à cette démarche lorsqu'elle vise de manière explicite des groupes ethniques ou raciaux ${ }^{6}$. Destinées en priorité ou exclusivement aux résidents des quartiers relevant de la

3. Voir Daniel Béhar, «Entre intégration des populations étrangères et politique de la ville: existe-t-il une discrimination positive à la française ?», Hommes et Migrations, $\mathrm{n}^{\circ} 1213$, juin 1998.

4. Fonds d'action et de soutien pour l'intégration et la lutte contre les discriminations.

5. La politique de la ville est loin d'épuiser le champ d'application des discriminations positives territoriales. Ferdinand Mélin-Soucramanien propose, par exemple, d'inclure dans ce registre les mesures dérogatoires du droit commun concernant les DOM-TOM, l'aménagement du territoire et la protection de l'environnement (cf. "Les adaptations du principe d'égalité à la diversité des territoires", Revue française de droit administratif, septembreoctobre 1997.

6. En ce sens, voir Gwénaële Calvès, "Affirmative action in French Law», La Revue Tocqueville/The Tocqueville Review, vol. XIX, n² 2, 1998; Véronique de Rudder et al., «Les enjeux politiques de lutte contre le racisme: discrimination justifiée, affirmative action, discrimination positive, parle-t-on de la même chose ? ", Les Cabiers du CIEREM, n 7, juin 2001. 
politique de la ville, certaines mesures d'accès à l'emploi ${ }^{7}$ laissent en effet supposer que la catégorie «quartiers prioritaires » a été délibérément conçue pour accorder un avantage spécifique à leurs habitants, notamment aux «jeunes issus de l'immigration», en compensation du racisme et de la discrimination dont ils sont victimes ${ }^{8}$.

La doctrine des pouvoirs publics met en exergue, quant à elle, les finalités très distinctes de la discrimination positive territoriale (cette terminologie n'étant pas forcément acceptée) et de l'affirmative action ${ }^{9}$. Cette dernière viserait l'égalité des résultats obtenus par les groupes ethniques (et les femmes) dans l'accès à certains "marchés » (universités, contrats publics, emplois privés), afin de corriger à la marge les conséquences d'un système social structurellement inégalitaire, et au prix d'une entorse grave aux principes méritocratiques et de cécité aux origines. La discrimination positive territoriale chercherait au contraire à restaurer l'égalité des chances entre les individus dans tous les aspects de la vie sociale, afin de redonner sens aux principes d'égalité et de mérite qui fondent le modèle républicain, mais demeurent trop abstraits étant donné les conditions d'existence réelles des «quartiers sensibles» et la prégnance des discriminations.

À l'examen des orientations de la politique de la ville, on peut se demander toutefois si sa finalité véritable n'est pas d'égaliser les résultats des groupes que sont les territoires plutôt que d'assurer l'égalité des chances des individus qui y résident. Ce pourrait être là un premier résultat paradoxal si l'on considère, contrairement à une lecture répandue en France ${ }^{10}$, que la fin ultime de l'affirmative action est une véritable égalité des chances entre les individus - obtenue par une

7. Par exemple: les quotas d'embauche d'habitants en contrepartie des exonérations accordées aux entreprises implantées dans les zones franches urbaines; les quotas plus ou moins explicites de jeunes des quartiers dans l'accès à différents programmes (emplois de ville aujourd'hui disparus, emplois-jeunes, TRACE, parrainage); le PACTE (Parcours d'accès aux carrières de la territoriale, de l'hospitalière et de l'État) récemment annoncé pour promouvoir l'accès de ces jeunes à la fonction publique.

8. Pour une analyse des ambiguïtés de la catégorie «jeunes des quartiers» et des insuffisances d'une stratégie territoriale de lutte contre les discriminations, voir Patrick Simon, «Les jeunes issus de l'immigration se cachent pour vieillir. Représentations sociales et catégories de l'action publique», VEI Enjeux, n²121, juin 2000.

9. Voir notamment: Conseil d'État, Sur le principe d'égalité, extrait du rapport public 1996, «Études et Documents», n 48, La Documentation française, 1996; Conseil national des villes, Rapport général 1994-1997, La Documentation française, 1997; Haut Conseil à l'intégration, Le Contrat et l'Intégration, rapport au Premier ministre, La Documentation française, 2004.

10. Voir par exemple Dominique Schnapper, La Relation à l'autre. Au cour de la pensée sociologique, Gallimard, 1998. 
compensation de la défaveur spécifiquement liée à l'appartenance à un groupe discriminé - plutôt qu'une égalité entre les groupes euxmêmes. D'un point de vue empirique, il est vrai que l'administration américaine supervisant la mise en ouvre de l'affirmative action se focalise sur la réduction des écarts entre groupes ethno-raciaux. Mais il faut rappeler que cette pratique n'a jamais été fondée sur la reconnaissance constitutionnelle de droits collectifs attribués aux minorités dans une optique «communautariste». La lecture qui prédomine en France perd de vue les débats complexes sur les justifications à long terme de l'affirmative action. On peut retenir celle que propose Ronald Dworkin: l'élimination des stéréotypes négatifs associés à l'identification ethno-raciale des individus et des discriminations diffuses qu'ils alimentent, sans laquelle l'égalité des chances entre lesdits individus ne 104 saurait être réelle ${ }^{11}$.

Outre la contestation de sa finalité, la littérature «grise» comme un grand nombre de commentateurs français attribuent deux principaux types d'effets pervers à la méthodologie de l'affirmative action. Inspirée de ses détracteurs néo-conservateurs américains, la première critique porte sur l'indexation de préférences sur des catégories ethno-raciales. Ce mécanisme est jugé à la fois restrictif (car trop lié à l'histoire particulière des Noirs) et inflationniste (car des minorités toujours plus nombreuses s'en prévaudraient), à la fois stigmatisant pour ses bénéficiaires et créateur de droits transitoires qu'ils ont tendance à considérer comme acquis, suscitant le ressentiment de ceux qui en sont privés et attisant la concurrence entre groupes ethniques, renforçant finalement les identifications communautaires, à l'encontre du but recherché ${ }^{12}$. Bref, le différentialisme juridique de l'affirmative action induirait une rupture dans l'égalité des droits et des chances, exacerbant la séparation des groupes au lieu de les rapprocher. Empruntée cette fois à ses contempteurs américains de gauche, une seconde critique porte sur l'effacement du politique au profit d'une action juridique essentiellement curative. L'affirmative action exonérerait l'État de ses responsabilités en matière de solidarité et conforterait une rhétorique de la victimisation chez ses bénéficiaires ${ }^{13}$.

11. Ronald Dworkin, A Matter of Principle, Cambridge, Mass., Harvard University Press, 1985. Pour une discussion et un approfondissement de la thèse de Dworkin, voir Daniel Sabbagh, L'Égalité par le droit. Les paradoxes de la discrimination positive aux États-Unis, Economica, «Études politiques», 2003.

12. Voir par exemple Dominique Schnapper, op. cit.

13. Voir Pierre Rosanvallon, La Nouvelle Question sociale. Repenser l'État-providence, Seuil, 1995. 
Consistant en une simple compensation juridique bénéficiant d'abord aux classes moyennes noires déjà intégrées, elle se montrerait incapable de transformer les structures sociales et urbaines créatrices d'inégalités ${ }^{14}$.

La méthode d'action de la politique de la ville devrait permettre, en principe, de contourner ces deux écueils. D'une part, en différenciant le traitement de territoires cibles plutôt que de populations cibles, cette politique préserverait le caractère universaliste des politiques sociales pour s'adresser à la diversité des habitants des quartiers plutôt qu'à tel ou tel groupe désigné par le critère illégitime des origines; au lieu de maintenir ces territoires dans un statut de dérogation permanente, leur traitement différencié par les services publics contribuerait à donner un contenu effectif au principe d'égalité; la politique de ville limiterait enfin les effets de stigmatisation, de ressentiment et de communautarisation en renforçant les liens entre tous les quartiers et habitants de la ville. D'autre part, à l'inverse de l'affirmative action, la politique de la ville afficherait une ambition proprement politique, celle d'une refondation locale de la solidarité. Bien qu'elle se présente comme une politique spécifique, elle n'a pas vocation à se substituer aux politiques du droit commun, ni à se cantonner dans un registre réparateur. En vertu de sa fonction incitative, elle doit contribuer à transformer les politiques ordinaires, c'est-à-dire à renforcer et adapter leurs moyens d'action sur les causes de l'exclusion. Puisqu'il s'agit de lutter contre toutes les inégalités au-delà des seuls effets des discriminations, les habitants des quartiers ne sont pas considérés comme des victimes justiciables d'une compensation, mais comme des individus injustement privés de la jouissance effective de leurs droits de citoyens.

On verra pourtant que les principales critiques adressées à la politique de la ville reviennent à pointer des inconvénients analogues, si on les transpose au plan territorial, à ceux de l'affirmative action: le différentialisme et la réparation. Eu égard à l'enjeu de la promotion socioéconomique des immigrés et de leurs descendants, la discrimination positive territoriale est confrontée à un paradoxe qui pourrait être aussi sa faiblesse majeure: sa finalité ultime - l'égalité de résultats entre territoires beaucoup plus que l'égalité des chances entre individus contraste fortement avec la finalité de l'affirmative action, sans pour autant qu'elle échappe aux inconvénients vus plus haut. Pour examiner

14. Voir Denis Lacorne, La Crise de l'identité américaine. Du melting pot au multiculturalisme, Fayard, 1997. 
cette hypothèse, il est nécessaire d'esquisser une généalogie de la politique de la ville, car ses priorités et méthodes ont évolué au fil de sa relativement courte histoire.

LA DISCRIMINATION POSITIVE ET LES SERVICES PUBLICS : LES LIMITES DE L'INCITATION

Dans le prolongement de la politique de «renouveau du service public», initiée par Michel Rocard en 1989 et placée sous le signe de l'équité, les premières références à la discrimination positive dans le discours de la politique de la ville sont repérables au début des années quatre-vingt-dix, à propos des services publics ${ }^{15}$. Évoquer la nécessité d'une discrimination positive revenait alors à inviter ceux-ci à se ren-

106 forcer, en quantité comme en qualité, dans les quartiers prioritaires ${ }^{16}$. Les contrats de ville signés pour la période 1994-1999 devaient inciter les services publics à remettre à niveau leur offre dans ces territoires et à ajuster leurs prestations aux besoins spécifiques des usagers. En conséquence, de nouveaux équipements ont été implantés et certaines améliorations ont été apportées aux conditions d'accueil et d'orientation. Mais l'essentiel des crédits de la politique de la ville a servi à financer des associations à dominante socio-culturelle et des dispositifs de médiation auxquels les services publics ont délégué de facto certaines compétences. L'objectif étant de «retisser le lien social», les financeurs se sont montrés assez peu regardants sur la qualité des prestations associatives. Quant aux médiateurs en tout genre (grands frères, agents d'ambiance, femmes relais, correspondants de nuit...), dont la politique de la ville a favorisé le spectaculaire essor, une lecture optimiste voudrait qu'ils aient contribué à rapprocher les usagers des services publics, pour aider les premiers à mieux faire usage de leurs droits et les seconds à repenser leurs prestations. Cependant, les recherches conduites sur le sujet montrent que ces médiations ont au moins autant été utilisées aux fins de pacifier leurs relations en faisant écran à des contacts trop frontaux ${ }^{17}$. La nature et la qualité des recrute-

15. Voir notamment les rapports de Jean-Marie Delarue, Banlieues en difficultés. La relégation, Syros, 1990, et de Paul Picard, L'Amélioration du service public dans les quartiers, rapport au ministre de la Ville, 1991.

16. L'Éducation nationale avait été pionnière, en la matière, avec l'institution des zones d'éducation prioritaires en 1981, mais la géographie des ZEP ne coïncide pas strictement avec celle de la politique de la ville.

17. Voir Éric Macé, «Les contours de la médiation: institution, conciliation, conformation. 
ments ont également soulevé des interrogations, la proximité des médiateurs avec la population des quartiers faisant jouer des considérations ethniques que le modèle républicain réprouve officiellement.

Derrière l'affichage d'un principe de discrimination positive, la politique de la ville aurait ainsi péché par excès de différentialisme, pour contribuer paradoxalement à l'avènement d'un «service public à deux vitesses ${ }^{18}$. Cette politique apparaît traversée par une ambiguïté constitutive, entre le besoin d'adapter les normes d'intervention des services publics pour tenir compte de la situation particulière des quartiers et la volonté d'y appliquer un traitement de «droit commun » afin d'éviter de les particulariser davantage. La norme appliquée aux quartiers tout au long des années quatre-vingt-dix a été celle de la "proximité», directement par l'implantation d'équipements ou indirectement par le recours à des formules associatives ou de médiation. Mais un excès de proximité risque d'accentuer le sentiment de captivité des habitants ${ }^{19}$, de renforcer leur identification au quartier au lieu de favoriser les liens avec d'autres citadins, voire d'aiguiser le ressentiment des populations limitrophes, jalouses de ces habitants perçus comme indûment privilégiés ${ }^{20}$.

La seconde critique majeure adressée aux contrats de ville, à la fin des années quatre-vingt-dix, portait sur leur registre réparateur, dépourvu d'impact sur les racines d'une ségrégation en partie imputable à l'action publique elle-même. Qu'il s'agisse de l'école, de l'emploi, des transports ou de l'habitat, c'est à l'échelle large des villes et des agglomérations que devait jouer la solidarité en faveur des quartiers défavorisés, sous l'impulsion d'une politique de la ville incitant l'action publique locale à infléchir ses règles d'allocation des ressources et à combattre les mécanismes de tri et d'éviction. Cette démarche d'agglomération a largement échoué. Sous l'égide des «sous-préfets à la ville»,

À propos d'un dispositif de “médiation” de la RATP », Revue française des affaires sociales, $\mathrm{n}^{\circ} 2$, avril-juin 1997; Sébastien Roché, «Les règles d'hospitalité et les professionnels de l'ordre en public », Les Cabiers du CR-DSU, n²2, mars 1999.

18. Sur cette question, voir Thomas Kirszbaum, «Services publics et fractures de la ville. La "pensée publique" entre diversité, éclatement et souci du rapprochement », Sociologie du travail, vol. 46, n², 2004.

19. Annie Maguer et Jean-Marc Berthet, Les Agents des services publics dans les quartiers difficiles, DGAFP, La Documentation française, 1997.

20. Daniel Béhar, Philippe Estèbe, Ville et Pauvreté. Connaissance scientifique et politiques publiques, Synthèses... Réflexions sur la connaissance des territoires urbains, DGUHC, 1999. 
l'État devait se faire «animateur » ${ }^{21}$, mais il n'a pu s'affirmer comme l'énonciateur d'une norme locale d'équité. Sur un plan strictement comptable, les «crédits spécifiques» de la politique de la ville se sont dans bien des cas substitués aux «crédits de droit commun» des administrations locales. Ces dernières ont fait quelquefois preuve d'activisme, mais le traitement différencié des territoires s'est opéré selon la rationalité propre à chaque institution, sans coïncidence méthodique avec la rationalité supposée de la géographie prioritaire de la politique de la ville ${ }^{22}$.

Les politiques de droit commun sont d'autant moins enclines à reconsidérer leurs priorités territoriales que l'idée de discrimination positive est apparue en dehors de toute demande sociale organisée. En dépit d'une rhétorique insistante sur la participation des habitants, la conception descendante (top-down) de l'effort prioritaire à accomplir en direction de ces quartiers interdit à leurs habitants d'en contrôler l'effectivité. Après quelques années de mise en œuvre des contrats de ville, un rapport officiel montrait ainsi que les situations de discrimination négative par les services publics étaient le cas de figure le plus répandu ${ }^{23}$. Il est néanmoins un domaine où la discrimination positive territoriale a été pratiquée avec succès: celui de l'amélioration du sort des fonctionnaires de l'État en poste dans les quartiers (par la valorisation des carrières, une nouvelle bonification indiciaire et une priorité de mutation). Mais cette discrimination positive là ne relevait pas d'un simple message incitatif. Elle était inscrite dans la loi du 26 juillet 1991.

La méthode n'était pas seule en cause dans la mobilisation incertaine des services publics. La finalité même de cette mobilisation n'était pas dépourvue d'ambiguité. À côté de l'égalité des chances, la politique de la ville a toujours énoncé une finalité concurrente, celle de la mixité des fonctions urbaines que traduit ce slogan qui a fait florès: "faire des quartiers comme les autres». Ici, la finalité de la discrimination positive territoriale n'est plus tant de questionner les modes d'organisation et de fonctionnement des services publics que d'homogénéiser la situation des territoires. Comme l'écrivent Jacques Donzelot et ses collègues, "ce qui compte, c'est la mise à disposition, en tous lieux,

21. Jacques Donzelot et Philippe Estèbe, L'État animateur. Essai sur la politique de la ville, Éditions Esprit, 1994.

22. Sur tous ces points, voir Philippe Estèbe, L'Usage des quartiers. Action publique et géographie dans la politique de la ville (1982-1999), L'Harmattan, 2004.

23. Jean-Pierre Sueur, Demain la ville, rapport au ministre de l'Emploi et de la Solidarité, 1998. 
d'une capacité d'éducation et de services la plus égale possible afin que nul ne puisse dire qu'il a subi une injustice dans le cadre de la République, ce n'est pas d'obtenir des résultats chiffrés de promotion de catégories ethniques par ailleurs ignorées dans le décompte des destinataires des services ${ }^{24}$ ».

\section{LE ZONAGE ÉCONOMIQUE :}

\section{LA DÉROGATION INSCRITE DANS LE DROIT}

La politique de la ville a fait un second usage de la discrimination positive territoriale, entendue cette fois comme un ensemble de mesures dérogatoires dont l'intensité varie selon le degré de handicap des «zones» où cette discrimination s'applique. Telle était la philosophie de la loi d'orientation pour l'aménagement et le développement du territoire (loi Pasqua du 4 février 1995) qui créait les zones urbaines sensibles (ZUS) et les zones de redynamisation urbaine (ZRU). La loi du 14 novembre 1996 mettant en œuvre le «pacte de relance pour la ville» ajoutait une catégorie supplémentaire: les zones franches urbaines (ZFU) qui allaient bénéficier du maximum d'avantages dérogatoires. À la différence des contrats de ville, le principe de discrimination positive était désormais inscrit dans le droit (sans toutefois que l'expression apparaisse formellement).

Le mécanisme de compensation des handicaps territoriaux cherchait à «assurer, à chaque citoyen, l'égalité des chances sur l'ensemble du territoire", en garantissant notamment "l'égal accès de chaque citoyen aux services publics» (art. $1^{\text {er }}$ de la loi du 4 février 1995). L'objectif visait tout autant les zones rurales qu'urbaines. Mais, dans les quartiers urbains, il s'agissait surtout de renforcer le volet économique de la politique de la ville en instaurant, pour une durée de cinq ans, un régime massif d'exonérations fiscales et sociales dans les zones franches urbaines. Le bénéfice de ces exonérations était subordonné à une clause d'embauche de $20 \%$ minimum de résidents de la zone (cette clause s'appliquant à partir de la troisième nouvelle embauche). Par ailleurs, une nouvelle forme de "contrats emploi consolidés» était créée: les «emplois de ville» réservés aux jeunes peu qualifiés (niveau bac ou inférieur) des ZUS.

Avec les quotas d'embauches dans les ZFU et le critère territorial

24. Jacques Donzelot et al., Faire société. La politique de la ville aux États-Unis et en France, Seuil, 2003, p. 128. 
de recrutement des emplois de ville, l'homologie formelle avec la méthode de l'affirmative action était plus forte que dans les contrats de ville. Le pacte de relance pour la ville est d'ailleurs le seul programme de la politique de la ville à avoir été présenté officiellement comme une démarche de discrimination positive, même si ses promoteurs ont pris soin de distinguer celle-ci de toute «logique communautaire» 25 . Rapidement, cependant, une série de rapports d'experts a fustigé cette méthode d'action, à partir d'arguments anti-affirmative action transposés à l'approche territoriale du pacte de relance ${ }^{26}$. La catégorie d'action retenue, fondée sur le découpage du territoire en zones, aurait reposé sur des critères arbitraires et vecteurs d'une inflation de ce que l'on peut appeler des «ayants droit territoriaux». La dérogation était pensée comme conjoncturelle, mais rien ne s'opposait à ce que la clas-

110 sification des quartiers selon le degré de leurs handicaps ne multiplie à l'infini le nombre de quartiers concernés (on en recensait déjà plus de 1300 contre une vingtaine aux origines de la politique de la ville), engendrant une demande sans fin de dérogation à la norme (de la part des élus locaux notamment) et éloignant la perspective du retour à un traitement égalitaire. Il était également reproché au zonage de susciter des «effets de frontière ", le découpage des zones à la rue près les isolant davantage du reste de la ville et suscitant une sorte de «discrimination à rebours» des habitants et entreprises exclus du champ de la dérogation.

Une seconde critique portait sur la compensation automatique des handicaps. Les contrats de ville avaient valeur d'engagement politique à mobiliser l'intervention publique à toutes les échelles territoriales, pour traiter non seulement les manifestations de la crise urbaine dans les quartiers, mais pour transformer aussi la structure sociale et urbaine à l'origine de cette crise. Appuyée sur la seule initiative des entreprises et faisant fi de la mobilisation des acteurs publics dans le cadre contractuel, la logique des zones franches aurait manifesté, au contraire, le renoncement des pouvoirs publics à agir sur les mécanismes producteurs d'exclusion dans les villes et agglomérations.

25. Voir Francis Idrac, «Le pacte de relance pour la ville», Regards sur l'actualité, n²22, juin 1996.

26. Voir Jean-Pierre Sueur, op. cit., ainsi que Jean-Paul Delevoye, Cohésion sociale et Territoires, Commissariat général du Plan, 1997; Conseil national des villes, Rapport général, 1997; Jacques Bravo, Rapport final de l'Instance d'évaluation de la politique de la ville en Îlede-France. Voir aussi Daniel Béhar et Philippe Estèbe, «Le pacte de relance pour la ville», Esprit, mars 1996. 
Au-delà des effets pervers du zonage, cette formule de discrimination positive territoriale pose question quant à sa finalité réelle. L'objectif d'égalisation des chances individuelles y tient finalement une place assez secondaire. Les «emplois de ville» suscitent en effet les mêmes interrogations que ces fonctions d'utilité sociale conçues au départ comme des sas temporaires d'insertion, mais qui contribuent bien souvent à enfermer ceux qui les occupent dans un statut permanent de personnes au seuil de l'emploi ordinaire. On peut également souligner le caractère fort peu contraignant de la clause d'embauche locale dans les ZFU et les lacunes en matière de contrôle du respect de leurs obligations par les employeurs, auxquels la loi de 1996 n'imposait d'ailleurs aucune exigence en termes de contrat et de durée du travail. En regard des avantages fiscaux et sociaux qui leur étaient consentis, indépendants pour la plupart de l'embauche d'habitants, le «traitement préférentiel» de ces derniers n'était qu'un aspect mineur $\mathrm{du}$ dispositif. D'autant que sa mise en ouvre locale s'est rarement accompagnée d'initiatives particulières tendant à réduire les distances sociales (et pas seulement géographiques) qui séparent les habitants des entreprises. Outre l'allègement des contraintes pesant sur ces dernières, dans une optique économique libérale, il semble que la finalité principale des zones franches ait été de «faire des quartiers comme les autres» en diversifiant les fonctions urbaines dans des quartiers exclusivement conçus autour de la fonction «habitat». Les responsables publics trouvaient cette vertu particulière au développement d'activités dans les quartiers: y faire venir des salariés de l'extérieur pour familiariser les habitants avec la valeur «travail».

Le zonage devait contribuer aussi à la mixité sociale puisqu'une des dérogations les plus significatives introduites par la loi de 1996 portait sur l'exonération, dans la géographie prioritaire, des surloyers acquittés par les ménages dépassant les plafonds de ressources en HLM. Credo officiel de la politique de la ville sous le gouvernement Juppé, la discrimination positive territoriale était donc loin d'avoir pour unique préoccupation d'assurer l'égalité des chances des habitants les moins favorisés. 


\section{LA TERRITORIALISATION \\ OU L'ÉCLIPSE DE LA DISCRIMINATION POSITIVE}

Associée à une philosophie politique «de droite» après l'alternance de $1993^{27}$, la discrimination positive est passée à la trappe du discours officiel sous le gouvernement Jospin. Depuis quelques années déjà, elle était l'objet d'une critique de gauche, celle visant les politiques spécifiques (territoriales ou non) destinées aux exclus et qui tendraient à les enfermer dans un statut d'exception stigmatisant ${ }^{28}$. C'est dans ce contexte que le ministère Aubry a envisagé un temps de renoncer à toute politique spécifique destinée aux quartiers. Mais cette position était difficilement tenable, vis-à-vis surtout des acteurs de terrain enga-

112 gés dans cette politique. Il fut donc décidé de mettre sur pied une commission, présidée par Jean-Pierre Sueur, chargée de dresser le bilan de la politique de la ville et de proposer des réorientations. Son rapport proposait l'abandon du concept de discrimination positive. Sur la base de son argumentaire anti-zones franches (inventées par la droite), leur extinction progressive fut décidée, tandis que les emplois de ville seraient absorbés dans les emplois-jeunes. Les contrats de ville (inventés par la gauche) étaient critiquables dans leur application plus que dans leur principe. Le principe de leur relance était acquis ${ }^{29}$, mais à condition d'en réviser la méthode et de trouver un nouveau concept mobilisateur. Dans l'air du temps depuis la décentralisation, celui de «territorialisation» s'est imposé, éclipsant toute référence à la discrimination positive dans le discours officiel.

La référence au territoire permettait de s'affranchir des limites du quartier ou de la zone. Au contraire, les contrats de ville de la «nouvelle génération» (2000-2006) déploieraient l'intervention publique sur une géographie souple et évolutive. L'idée d'en «faire plus pour ceux qui ont moins » restait d'actualité, mais l'affichage d'un principe de territorialisation évitait de véhiculer cette idée que l'on en faisait plus pour certaines populations - immigrées ou supposées

27. Cf. les violentes polémiques qui ont suivi en 1994 la publication du rapport Minc, La France de l'an 2000 pour le Commissariat général du Plan (1994). Ce rapport, qui devait servir de plate-forme électorale au candidat Balladur, semblait opposer l'équité à l'aspiration même à l'égalité.

28. Voir par exemple Robert Castel, «Les pièges de l'exclusion», Lien social et Politiques, $\mathrm{n}^{\circ} 34,1995$.

29. Le rapport proposait de les intituler «contrats d'agglomération». 
telles - que pour d'autres. Parler de territoires plutôt que de quartiers ou de zones signifiait que l'intervention publique ne devait plus obéir à la seule exigence de proximité physique avec les habitants, mais devait insérer ceux-ci et leurs territoires dans le monde des flux urbains. Il fallait pour cela concevoir des stratégies «d'accessibilité» aux divers marchés urbains (éducation, logements, santé, transports, formation, emploi, culture...). À cette fin, les contrats de ville devaient assurer la traduction territoriale de la loi de lutte contre les exclusions du 29 juillet 1998. Fixant «un objectif général de retour au droit commun", cette loi s'attachait à "préciser les modalités d'accès à des droits déjà reconnus de façon à les rendre effectifs» sans construire de «systèmes spécifiques de prise en charge» des exclus, se félicitait la commission Cavallier chargée de préciser la méthode des futurs contrats de ville ${ }^{30}$.

Censés aiguillonner le processus de territorialisation des politiques publiques concernées par l'enjeu des quartiers en difficulté, ces contrats de ville devaient sortir du registre curatif en mobilisant les politiques de droit commun au niveau «structurant» des agglomérations. L'idée n'était pas nouvelle, mais la refonte du paysage territorial résultant des lois Voynet, Chevènement et Gayssot-Besson, adoptées à la fin des années quatre-vingt-dix ${ }^{31}$, devait aider la politique de la ville à sortir de son «ghetto". À l'échelle des régions et des agglomérations, les contrats de ville ouvriraient des scènes locales permettant aux politiques de droit commun de se coordonner pour renforcer leurs moyens et adapter leurs modes d'action aux enjeux de la solidarité urbaine. La territorialisation visait à inscrire dans l'action publique ordinaire le principe d'un traitement différencié des territoires - que cela passe par un «plus» ou par un «mieux» - au lieu de déroger au droit commun pour le laisser fonctionner à l'identique et faire porter à une politique exceptionnelle unique la responsabilité de cette solidarité. Comme le précisait la circulaire du Premier ministre relative aux contrats de ville 2000-2006, «la politique de la ville n'est pas une politique sectorielle de plus, mais une dimension de toutes les politiques publiques».

30. Georges Cavallier, Nouvelles Recommandations pour la négociation des contrats de ville de la nouvelle génération (2000-2006), rapport au ministre délégué à la Ville, 1999.

31. Il s'agit respectivement de la loi du 25 juin 1999 pour l'aménagement et le développement durable du territoire, de la loi du 12 juillet 1999 relative au renforcement et à la simplification de la coopération intercommunale et de la loi sur la solidarité et le renouvellement urbain du 13 décembre 2000. 
À vouloir éliminer les inconvénients de la discrimination positive, la démarche de territorialisation prenait le risque de tomber dans l'excès inverse, celui de la banalisation d'une politique exceptionnelle.

Pas plus que les anciens contrats de ville, les nouveaux n'ont eu valeur d'engagement formel à accomplir un effort prioritaire en direction des quartiers défavorisés? Sans même parler d'obligations contractuelles, le rapport que la Cour des comptes a consacré en 2002 à la politique de la ville a relevé l'absence quasi générale d'objectifs de résultats dans ces contrats. À défaut, des objectifs de moyens sont quelquefois définis, mais le rapport les a jugés flous et trop peu contraignants. Dans la plupart des cas, ce sont des listes non hiérarchisées d'actions à conduire dans tous les domaines couverts par la politique de la ville qui tiennent lieu d'engagements. Le rapport a éga-

114 lement relevé l'impossibilité pratique d'identifier d'éventuels mécanismes de discrimination positive (ou négative). Dans l'acception minimaliste que la Cour des comptes donne à cette notion, le moins serait de mesurer la réalité des «crédits de commun» censé abonder les «crédits spécifiques» de la politique de la ville ${ }^{32}$.

\section{ÉGALISER LES RÉSULTATS DES TERRITOIRES \\ PLUTÔT QUE LES CHANCES DES HABITANTS}

Sans attendre les résultats plus précis des évaluations locales, à mi-parcours des contrats de ville, le nouveau ministre de la Ville, Jean-Louis Borloo, a conclu à leur échec. Dans un contexte où l'idée de discrimination positive est remise à l'honneur par une partie de la droite, la loi d'orientation et de programmation pour la ville et la rénovation urbaine ( $1^{\text {er }}$ août 2003) semble fournir une assise législative au principe de discrimination positive territoriale s'agissant du traitement des zones urbaines sensibles par les politiques de droit commun. La loi énonce un «objectif de réduction progressive des écarts constatés avec les autres villes ou quartiers, et de retour au droit commun» qu'elle décline thème par thème (emploi, habitat, santé, éducation...). Elle établit à cette fin une liste nationale «d'indicateurs de résultats» relatifs à l'évolution des ZUS et «d'indicateurs de moyens» relatifs à chaque politique publique concernée. En réalité, la loi ne fait que créer les conditions d'une mise en regard parallèle de l'évolution des ZUS et des

32. Cour des comptes, La Politique de la ville, rapport public particulier, février 2002. 
moyens engagés, lesquels ne comportent aucun élément de comparaison avec ceux engagés en faveur d'autres territoires que les ZUS. Les indicateurs doivent alimenter les travaux d'un Observatoire national des ZUS qui feront l'objet d'un débat parlementaire, mais ils n'impliquent en eux-mêmes aucune obligation de «faire plus pour ceux qui ont moins».

Localement, la loi Borloo invite l'État et ses partenaires à élaborer des «programmes d'action» qui fixent, pour chaque zone et sur une période de cinq ans, des objectifs de résultats chiffrés «en vue de réduire les inégalités sociales et les écarts de développement entre les territoires». Ces objectifs de résultats n'impliquent pas davantage une obligation de moyens. La loi est d'ailleurs singulièrement muette sur ce point. Les seuls moyens «spécifiques» existants, en diminution régulière depuis deux ans, sont ceux des contrats de ville. Or, ces derniers, tout comme les trois lois précitées organisant la nouvelle gouvernance des territoires, sont passés sous silence. La loi n'invite à aucun débat local et contradictoire sur l'effectivité de la mobilisation des politiques publiques. Elle se situe à nouveau dans le registre de l'incitation, faisant le pari d'un entraînement mécanique du savoir (celui que produiront les indicateurs collectés localement et transmis à l'Observatoire national) sur l'action ${ }^{33}$.

$\mathrm{Si}$, en dépit d'un affichage volontariste, le renforcement du volet social de la politique de la ville demeure très aléatoire, il en va tout autrement du développement économique et des actions de rénovation urbaine. La création de 41 nouvelles zones franches urbaines et la prolongation des 44 premières jusqu'en 2008 (donnant raison à ceux qui dénonçaient la pérennisation de l'exception) impliquent ici une véritable obligation de moyens. De même, les crédits de l'État affectés aux opérations urbaines de grande ampleur, prévues par la loi, sont «sanctuarisés» jusqu'en 2008. Ces deux programmes mobilisent des moyens financiers autrement plus conséquents que le volet social de la politique de la ville. Ils confirment et accentuent la primauté accordée à l'objectif de mixité urbaine sur celui d'égalité individuelle des chances.

La mixité urbaine doit contribuer à la banalisation des quartiers en

33. Pour une analyse critique de la loi Borloo, voir Philippe Méjean, «La politique de la ville à l'épreuve de la loi Borloo ", Études foncières, n 106, novembre-décembre 2003; Renaud Epstein, «La loi Borloo: renforcement ou remplacement de la politique de la ville ?», Revue de droit sanitaire et social, à paraître. 
les dotant de la diversité des attributs constitutifs d'un mode de vie urbain «normal»: des habitants divers par leurs origines sociales et ethniques, un habitat mixte quant à son statut (public et privé) et la taille des logements (pour éviter la sur-représentation des familles nombreuses), une variété d'activités (entreprises, commerces, services publics...) donnant des raisons de venir aux citadins de l'extérieur. L'objectif de «réduction des écarts» et de «retour au droit commun», affiché par la loi Borloo et par des textes antérieurs, s'inscrit clairement dans cette ligne ${ }^{34}$. Cette formule de discrimination positive territoriale s'attache à réaliser une égalité de résultats entre territoires plutôt que l'égalité des chances entre habitants. Les deux finalités sont d'ailleurs potentiellement contradictoires: le territoire ne risque-t-il pas de s'appauvrir (statistiquement) par l'évaporation de ses «meilleurs» éléments

116 si l'action publique leur fournit les ressources nécessaires pour retrouver le chemin d'une mobilité résidentielle ascendante? Plutôt que d'aider les habitants à accéder à différents marchés urbains, la priorité centrale des pouvoirs publics est la distribution harmonieuse des pauvres et des immigrés dans le tissu urbain. En témoigne la complémentarité des deux types de quotas validés par la loi: l'un vise à créer $20 \%$ de logements sociaux dans toutes les villes; l'autre permet de refuser l'attribution d'un logement social dans un quartier de la politique de la ville, au nom de la mixité sociale ${ }^{35}$.

À regarder l'agencement de ses priorités, la politique de la ville semble considérer que la mixité sociale dans l'espace résidentiel est une condition nécessaire, voire suffisante, de l'égalité réelle des chances. Ce n'est pourtant pas l'objectif d'égalité des chances qui donne à la mixité urbaine un rang aussi prépondérant dans l'ordre des préoccupations de la politique de la ville, mais la lutte contre le «ghetto». Depuis la loi d'orientation pour la ville (1991), officiellement présentée comme une «loi anti-ghettos», ce terme fonctionne comme un code servant à désigner les regroupements résidentiels d'immigrés (ou supposés tels) et signifier l'échec de leur intégration ou le danger de «dérives commu-

34. Plusieurs dispositifs promus par la gauche reflétaient déjà cette orientation: dès les années 1980, avec «Banlieues 89», qu'animaient des architectes en promettant «d'en finir avec les grands ensembles", puis à travers la loi d'orientation pour la ville (1991), les grands projets urbains (1992), les grands projets de ville (1999) et la loi solidarité et renouvellement urbains (2000). Le pacte de relance pour la ville, adopté par la droite en 1996, faisait aussi de la mixité urbaine l'une de ses priorités.

35. Voir Patrick Simon et Thomas Kirszbaum, Les Discriminations raciales et ethniques dans l'accès au logement social, GELD, 2001, note $\mathrm{n}^{\circ} 3$. 
nautaires ». Comment accorder un avantage préférentiel sur une base territoriale à des populations, quand on affiche dans le même temps l'intention de mettre fin à l'anomalie que constitue leur regroupement sur les territoires qui donneraient droit à cet avantage ? L'urbain et le développement économique seraient-ils les seuls moyens de rendre politiquement et socialement acceptable une action préférentielle sur certains secteurs de la ville? Serait-il concevable que les mêmes montants soient affectés à des dépenses sociales destinées à des personnes d'origine immigrée?

Les responsables publics français ont raison d'insister sur les dissemblances entre la discrimination positive territoriale et l'affirmative action américaine. Mais le contraste entre leurs finalités respectives tient sans doute moins à l'opposition entre l'égalité des résultats et l'égalité des chances qu'à la nature du «sujet de l'égalité » dans les deux politiques ${ }^{36}$. L'affirmative action vise la promotion socio-économique de certaines catégories d'individus identifiés par leur appartenance à un groupe ethno-racial ou sexuel, même si, avec la bureaucratisation progressive de cette politique, le critère d'appartenance au groupe a paru changer le sens de sa finalité au profit de la promotion du groupe lui-même ${ }^{37}$. Le sujet de l'égalité dans la discrimination positive territoriale est le territoire lui-même, davantage que les individus qui le composent. Cette prévalence du territoire n'a fait que se renforcer depuis qu'existe la politique de la ville ${ }^{38}$, sans doute en raison de la politisation de la question de l'immigration, en France, depuis vingt ans.

L'ironie de la discrimination positive territoriale est sans doute la suivante: sans procurer des avantages individuels aussi substantiels que l'affirmative action, sa méthode n'échappe pas aux effets pervers (différentialisme, réparation) généralement associés à celle-ci. La finalité ambiguë et l'efficacité incertaine de la politique de la ville ne sont pas de nature à renforcer la crédibilité du message républicain en direc-

\footnotetext{
36. Sur la notion de sujets de l'égalité, voir Douglas Rae et al., Equalities, Cambridge, Mass., Harvard University Press, 1981.

37. Voir John D. Skrentny, The Ironies of Affirmative Action: Politics, Culture, and Justice in America, Chicago, University of Chicago Press, 1996.

38. Voir Jacques Donzelot et al., op. cit.
} 
tion des populations issues de l'immigration, alors même que cette discrimination positive «à la française» sert souvent de justification au refus d'importer l'affirmative action dans notre pays.

L'efficacité de la politique de la ville en matière de promotion socio-économique des immigrés et de leurs descendants peut être interrogée. La finalité principale de cette formule de "discrimination positive territoriale» est l'égalité de résultats entre territoires plutôt que l'égalité des chances entre habitants. Paradoxalement, sans procurer des avantages individuels aussi substantiels que l'affirmative action, sa méthode n'échappe pas aux effets pervers (différentialisme, réparation) associés à cette dernière. 